\title{
2-氨基-4,5-二取代烃嘧啶类衍生物的新合成方法
}

\author{
邓兰青*, $a$ 徐中海 ${ }^{a}$ 尚云峰 ${ }^{a}$ 戴文静 ${ }^{a}$ 胡辉敏 ${ }^{a}$ \\ 邓穗平 $b$ 欧阳健明 $b$ \\ ( ${ }^{a}$ 岳阳职业技术学院 岳阳市中草药综合利用重点实验室 岳阳 414000) \\ ( ${ }^{b}$ 暨南大学生物矿化与结石病防治研究所 广州 510632)
}

\begin{abstract}
摘要 研究了 2-氨基-4,5-二烃基取代嘧啶类衍生物的一个新的简易合成方法，该方法以简单且廉价的 4 个芳香醛和 3 个脂肪醛为原料, 经过四步反应得到 12 个 2-氨基- 4,5 -二烃基取代嘧啶类化合物，总收率在 40\% 70\%, 经氢核磁共振 $\left({ }^{1} \mathrm{H}\right.$ NMR)、碳核磁共振 $\left({ }^{13} \mathrm{C}\right.$ NMR $)$ 和高效液质联用(LC-MS)对其目标产物进行表征.
\end{abstract}

关键词 2-氨基嘧啶; 芳香醛; 脂肪醛; 合成性

\section{A New Synthesis of 2-Amino-4,5-substituted Pyrimidine Derivatives}

\author{
Deng, Lanqing ${ }^{*, a}$ \\ Xu, Zhonghai ${ }^{a}$ \\ Shang, Yunfeng ${ }^{a}$ \\ Dai, Wenjing ${ }^{a}$ \\ Hu, Huimin ${ }^{a}$ \\ Deng, Suiping ${ }^{b}$ \\ Ouyang, Jianming ${ }^{b}$ \\ (Key Laboratory of Yueyang Chinese Herbal Utilization, Yueyang Vocational Technical College, Yueyang 414000) \\ (Institute of Biomineralization and Lithiasis Research, Jinan University, Guangzhou 510632)
}

\begin{abstract}
In this paper, a novel synthetic method for 2-amino-4,5-substituted pyrimidine derivatives was reported, which began from cheap and simple four aromatic aldehydes and three aliphatic aldehydes. 12 compounds of 2-amino-4,5-substituted pyrimidine derivatives were synthesized by four steps with yields of $40 \% \sim 70 \%$. The structures of terminational compounds were determined by ${ }^{1} \mathrm{H}$ NMR, ${ }^{13} \mathrm{C}$ NMR and LC-MS techniques.
\end{abstract}

Keywords 2-amino-pyrimidine; aromatic aldehyde; aliphatic aldehyde; synthesis

嘧啶是一类非常重要的杂环化合物, 广泛应用于医 药领域. 大量研究表明该类化合物具有较好的生物活 性, 如杀虫、杀菌、除草、抗病毒、抗癌等 ${ }^{[1 \sim 3]}$. 作为重 要生物分子 DNA 和 ATP 的结构单元, 嘧啶并环类化合 物具有多种生理活性, 是新药设计中的优势结构, 许多 已上市及临床阶段的药物分子中都含有该结构单元. 该 类化合物具有广泛的药理活性, 是一类重要的蛋白激酶 抑制剂, 在临床上广泛用于肿瘤、炎症、心血管、哮喘 及神经退行性等多种疾病的治疗. 2001 年 Bridges 对蛋 白激酶 C、酪氨酸蛋白激酶、丝裂原活化蛋白激酶等蛋 白激酶抑制剂的研究进展进行了综述, 其中很多抑制剂 分子都属于嘧啶及嘧啶并环类化合物 ${ }^{[4]}$.

Pranab 等 ${ }^{[5]}$ 研究了从 1,1-二甲氧基-4,4-二(甲硫基)3-丁烯-2-酮与硝酸胍在甲醇钠或乙醇钠的甲醇或乙醇 溶液中, 加热回流, 即可得到嘧啶环化合物(Scheme 1),
其产率为 $65 \%$ 和 $60 \%$.<smiles>[R]Oc1cc(C(C)OC)nc([Y])n1</smiles>

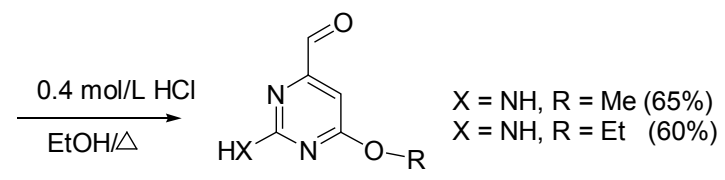

\section{Scheme 1}

Deshmukh 等 ${ }^{[6]}$ 研究了嘧啶的关环反应, 均采用 1 个 当量芳香醛 $(10 \mathrm{mmol})$ 、氧基乙酸乙酯 $(10 \mathrm{mmol})$ 和 $\mathrm{NaOH}(10 \mathrm{mmol})$ 在溶于乙醇中, 搅拌 $10 \mathrm{~min}$, 然后加入 盐酸胍, 加热至回流, TLC 跟踪反应, 待反应结束. 将反 应后的混合液倒入 $10 \mathrm{mmol} \mathrm{HCl}$ 的冰水混合液中, 摚拌,

* E-mail: denglq1111@126.com

Received September 22, 2011; revised December 20, 2011; published online February 14, 2012

Project supported by the National Natural Science Foundation of China (No. 30672103) and the Fundation of Hunan Educational Committee (No. 10C0336).

国家自然科学基金(No. 30672103)和湖南省教育厅(No. 10C0336)资助项目. 
过滤后得到目标产物, 其合成线路如 Eq. 1 所示.

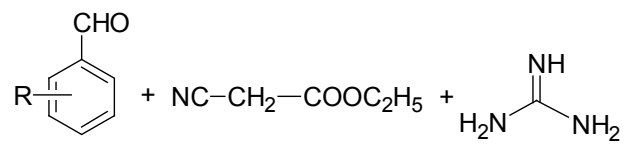<smiles>[R]c1ccc(-c2nc(N)nc(O)c2C#N)cc1</smiles>

Takahashi 等 ${ }^{[7]}$ 研究了在碘和碳酸钾或是 1,3 -二碘5,5-二甲基海因(DIH) 存在下, 多种芳香醛和脂肪醛与 1,3-丙二胺反应，能高效率地转化为 $1,4,5,6$-四氢嘧啶, 1,4,5,6-四氢嘧啶在二氧化锰的催化下, 以中等产率 生成 2-取代嘧啶类化合物(Scheme 2).

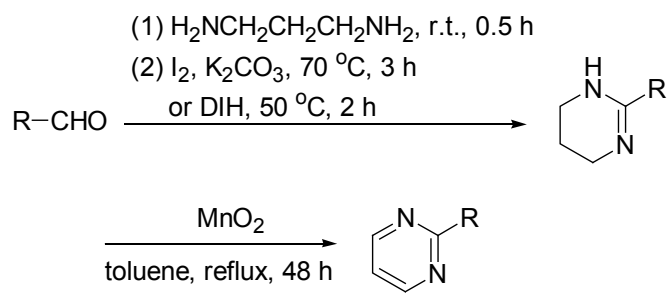

\section{Scheme 2}

我们在研究抗肿瘤激酶抑制剂的过程中设计了一 系列 2-氨基嘧啶类衍生物, 本文作者以廉价苯甲醛类衍 生物(4 个)为原料, 如 Scheme 3 所示, 整个反应总收率 在 $40 \% \sim 70 \%$. 第一步, 1a 1d 分别与对甲基苯磺酰腙 反应生成苯磺酰腙化合物 $\mathbf{2}$; 第二步, 化合物 2 分别与 脂肪醛 $3 \mathbf{a} \sim 3 \mathbf{c}$ 反应回流，形成 12 个烷基芳香酮 $\mathbf{4}^{[8,9,10] \text {; }}$
第三步，烷基芳香酮 4 在 $N, N$-二甲基甲酰胺二甲基缩醛 的 DMF 溶液中回流; 第四步，在甲醇钠碱性条件下，与 盐酸胍反应关嘧啶环.

\section{1 实验部分}

\section{2 试剂与仪器}

WFH-203 型三用紫外分析仪; 核磁共振谱由 Bruker-AV400 型核磁共振仪测定; 色谱仪由美国安捷 伦 Aglilent 1100 高效液相色谱仪测定; 液质联用仪由美 国安捷伦 Aglilent 6000LC/MS 测定. 乙醇、四氢呋喃均 为无水分析纯试剂; 其它试剂均为市售分析纯试剂.

\section{2 苯腙类化合物 2 的合成}

称取 $1.0 \mathrm{mmol}$ 化合物 $\mathbf{1}(\mathbf{1 a} \sim \mathbf{1 d})$ 分别置于 $50.0 \mathrm{~mL}$ 的圆底烧瓶中, 加入 $15.0 \mathrm{~mL}$ 无水四氢呋喃, 在 $25{ }^{\circ} \mathrm{C}$ 下 摚拌, 将 $1.0 \mathrm{mmol}$ 的甲苯磺酰腙分别加入上述反应瓶 中，反应 $2 \mathrm{~h}$ ，反应结束后，真空旋干. HPLC 检测产率均 在 $90 \%$ 以上. 得到的 4 个中间体 2 , 无需提取分离, 直接 用作下一步反应.

\section{3 烷基芳香酮化合物 4 的合成}

Angle 等 ${ }^{[10,11]}$ 研究了烷基芳香酮的合成, 其合成路 线如 Eq. 2, 采用 1 equiv.烷基醛、2 equiv. 苯甲醛磺酸腙、 2 equiv.的乙醇钠的乙醇溶液, 三者混合，搅拌，直至反 应液的粉红色退去, 结束反应. Angle 课题组 ${ }^{[10]}$ 采用此 方法合成出 11 种䣯。

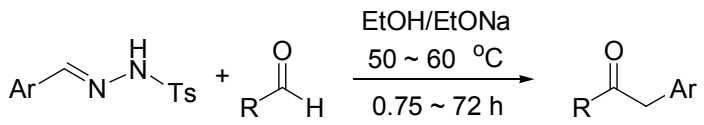

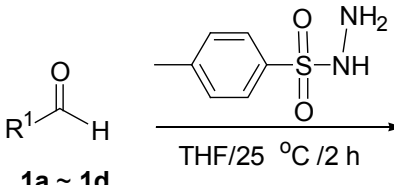<smiles>[R]C(=O)C([R])=CN(C)C</smiles>

5<smiles>[R]C=NNS(=O)(=O)c1ccc(C)cc1</smiles>

2

$80{ }^{\circ} \mathrm{C} / 18 \mathrm{~h}$

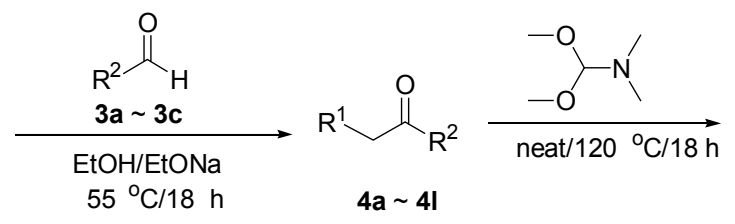

$$
6 a \sim 6 l
$$

1a<smiles>O=Cc1ccc(OC(F)(F)F)cc1</smiles>

$3 a$<smiles>CCC(C)CC=O</smiles>

$1 \mathrm{~b}$<smiles>O=Cc1ccc(C(F)(F)F)cc1</smiles>

1c<smiles>O=Cc1ccc(Cl)cc1</smiles>

1d<smiles>O=Cc1ccc(Cl)c(F)c1</smiles> 
我们课题组按照 Angle 课题组合成方法合成烷基芳 香酮. 新制 $20 \mathrm{mmol} / \mathrm{L}$ 乙醇钠-乙醇溶液; 将得到的中间 体 2 每个分成三等份(附上标签 $1 a \_3 a, 1 a \_3 b^{[11]}, 1 a \_3 c$, $1 b \_3 a, 1 b \_3 b^{[11]}, 1 b \_3 c, 1 c \_3 a, 1 c \_3 b, 1 c \_3 c, 1 d \_3 a$, $\left.1 d \_3 b, 1 d \_3 c\right)$, 分装在 12 个 $10 \mathrm{~mL}$ 圆底烧瓶中, 并在瓶 身外附上一层铝䇴纸, 以达到避光作用; 在避光条件下 分别依次加入无水乙醇 $1.5 \mathrm{~mL}$ 、相对应的 $0.15 \mathrm{mmol}$ 醛 类化合物 3, $1.5 \mathrm{~mL}$ 乙醇钠溶液, 密封, $55{ }^{\circ} \mathrm{C}$ 下回流 $18 \mathrm{~h}$. 反应后, 真空旋干, 萃取 (二氯甲烷/水), 萃取过程用 TLC 跟踪监测, 旋干后得到中间体 4. 我们从 12 个中间 体 4 中抽取 3 个(1a_3b, 1b_3a, 1c_3c), 经气相色谱分离 $\left(t_{\mathrm{R} 1 \mathrm{a} \_3 \mathrm{~b}}=21.85, t_{\mathrm{R} 1 \mathrm{~b}_{3} \mathrm{a}}=20.85, t_{\mathrm{R} 1 \mathrm{a} 3 \mathrm{~b}}=20.15\right)$. 化合物 $\mathbf{4}_{\mathrm{a}_{\mathrm{a}} 3 \mathrm{~b}},{ }^{1} \mathrm{H} \mathrm{NMR}\left(\mathrm{CD}_{3} \mathrm{OD}, 400 \mathrm{MHz}\right) \delta: 1.13(\mathrm{~d}, J=6 \mathrm{~Hz}$, $6 \mathrm{H}), 2.05 \sim 2.10(\mathrm{~m}, 1 \mathrm{H}), 2.52(\mathrm{~d}, J=13 \mathrm{~Hz}, 2 \mathrm{H}), 3.37$ (s, $2 \mathrm{H}), 7.23(\mathrm{~d}, J=8 \mathrm{~Hz}, 2 \mathrm{H}), 7.41(\mathrm{~d}, J=8 \mathrm{~Hz}, 2 \mathrm{H})$; 化合 物 4 $4_{1 \mathrm{~b} \_3 \mathrm{a},}{ }^{1} \mathrm{H}$ NMR $\left(\mathrm{CD}_{3} \mathrm{OD}, 400 \mathrm{MHz}\right) \delta: 0.91(\mathrm{t}, J=6 \mathrm{~Hz}$, $3 \mathrm{H}), 1.07(\mathrm{~d}, J=6 \mathrm{~Hz}, 3 \mathrm{H}), 1.42 \sim 1.48(\mathrm{~m}, 2 \mathrm{H}), 1.93 \sim$ $2.00(\mathrm{~m}, 1 \mathrm{H}), 2.47(\mathrm{~d}, J=8 \mathrm{~Hz}, 2 \mathrm{H}), 3.52(\mathrm{~s}, 2 \mathrm{H}), 7.25(\mathrm{~d}$, $J=8 \mathrm{~Hz}, 2 \mathrm{H}), 7.63(\mathrm{~d}, J=8 \mathrm{~Hz}, 2 \mathrm{H})$; 化合物 $\mathbf{4}_{1 \mathrm{c} \_3 \mathrm{c},}{ }^{1} \mathrm{H}$ NMR $\left(\mathrm{CD}_{3} \mathrm{OD}, 400 \mathrm{MHz}\right) \delta: 0.96(\mathrm{t}, J=6 \mathrm{~Hz}, 3 \mathrm{H}), 1.67 \sim$ $1.72(\mathrm{~m}, 2 \mathrm{H}), 2.47(\mathrm{t}, J=6 \mathrm{~Hz}, 2 \mathrm{H}), 3.52(\mathrm{~s}, 2 \mathrm{H}), 7.35(\mathrm{~d}$, $J=8 \mathrm{~Hz}, 2 \mathrm{H}), 7.46(\mathrm{~d}, J=8 \mathrm{~Hz}, 2 \mathrm{H})$.

本课题组将上述分离纯化后的 3 个烷基芳香酮 (1a_3b, 1b_3a, 1c_3c)和未经纯化的 3 个相同烷基芳香酮 作个对比, 经后续反应合成至最终目标产物后, 测其产 率, 发现纯化和未纯化的最终产率相当. 为了降低成本, 我们选择不分离纯化, 直接将中间体 4 用作下一步反应.

\section{4 中间体 1-二甲胺-2-芳基烯酮(5)的制备}

采用 neat 反应, 将 12 个中间体 4 分别放置于 $4 \mathrm{~mL}$ 的反应瓶中, 加入 $1.0 \mathrm{~mL}$ DMF-二甲基缩醛溶液, 密封, 加热至 $120{ }^{\circ} \mathrm{C}$ 回流, 反应 $18 \mathrm{~h}$, 直到反应结束, 真空旋 干，得到 12 个黄色油状中间体 $\mathbf{5}$.

\section{5 化合物 6 的制备}

新配备 $0.3 \mathrm{~mol} / \mathrm{L}$ 胍的无水乙醇溶液和 $0.3 \mathrm{~mol} / \mathrm{L}$ 甲 醇钠-甲醇溶液, 在 $5 \mathrm{~mL}$ 的反应瓶中, 依次加入中间体 $5,1.0 \mathrm{~mL} 0.3 \mathrm{~mol} / \mathrm{L}$ 胍的无水乙醇溶液、 $1.0 \mathrm{~mL} 0.3 \mathrm{~mol} / \mathrm{L}$ 甲醇钠-甲醇溶液, 密封, 加热至回流, 反应 $18 \mathrm{~h}$. 反应 后的产物经过制备色谱分离得到 12 个目标化合物 6a 61.

4-(2-甲基丁基)-5-[4-(三氟甲氧基)苯基]嘧啶-2-胺 (6a): $13.0 \mathrm{mg}$, 产率 40\%, LC-MS 分析纯度 96.3\%, m.p. 597.74, 分子式 $\mathrm{C}_{16} \mathrm{H}_{18} \mathrm{~F}_{3} \mathrm{~N}_{3} \mathrm{O} .{ }^{1} \mathrm{H}$ NMR $\left(\mathrm{CD}_{3} \mathrm{OD}, 400\right.$ MHz) $\delta: 0.96\left(\mathrm{t}, J=6 \mathrm{~Hz}, 3 \mathrm{H}, \mathrm{CH}_{3}\right), 1.07(\mathrm{~d}, J=10 \mathrm{~Hz}$, $\left.3 \mathrm{H}, \mathrm{CH}_{3}\right), 1.55 \sim 1.60\left(\mathrm{~m}, 2 \mathrm{H}, \mathrm{CH}_{2}\right), 2.04 \sim 2.11(\mathrm{~m}, 1 \mathrm{H}$, $\mathrm{CH}), 2.51\left(\mathrm{~d}, J=8 \mathrm{~Hz}, 2 \mathrm{H}, \mathrm{CH}_{2}\right), 7.03(\mathrm{~d}, J=8 \mathrm{~Hz}, 2 \mathrm{H}$, benzene), $7.68(\mathrm{~d}, J=8 \mathrm{~Hz}, 2 \mathrm{H}$, benzene), $8.90(\mathrm{~s}, 1 \mathrm{H}$, pyrimidine); ${ }^{13} \mathrm{C}$ NMR (DMSO- $d_{6}$ ) $\delta: 165.54,158.32$, $150.65,148.24,131.63,127.45,123.62,120.35,113.53$, 41.47, 35.85, 31.73, 22.54, 10.68; ESI-MS $m / z$ (\%): 326 $\left([\mathrm{M}+1]^{+}, 100\right)$.

4-异丁基-5-[4-(三氟甲氧基)苯基]嘧啶-2-胺 (6b): $18.6 \mathrm{mg}$, 产率 $60 \%$, LC-MS 分析纯度 $97.2 \%$, m.p. 586.47, 分子式 $\mathrm{C}_{15} \mathrm{H}_{16} \mathrm{~F}_{3} \mathrm{~N}_{3} \mathrm{O} .{ }^{1} \mathrm{H}$ NMR $\left(\mathrm{CD}_{3} \mathrm{OD}, 400\right.$ MHz) $\delta: 0.91\left(\mathrm{~d}, J=6 \mathrm{~Hz}, 6 \mathrm{H}, \mathrm{CH}_{3}\right), 1.82 \sim 1.87(\mathrm{~m}, 1 \mathrm{H}$, $\mathrm{CH}), 2.51\left(\mathrm{~d}, J=8 \mathrm{~Hz}, 2 \mathrm{H}, \mathrm{CH}_{2}\right), 7.05(\mathrm{~d}, J=8 \mathrm{~Hz}, 2 \mathrm{H}$, benzene), 7.68 (d, $J=8 \mathrm{~Hz}, 2 \mathrm{H}$, benzene), 8.85 (s, $1 \mathrm{H}$, pyrimidine); ${ }^{13} \mathrm{C}$ NMR (DMSO- $\left.d_{6}, 100 \mathrm{MHz}\right) \delta: 169.58$, $159.97,150.83$, 149.24, 127.24, 126.43, 125.50, 125.62, 120.36, 115.31, 41.64, 29.84, 22.57; ESI-MS $m / z$ (\%): 312 $\left([\mathrm{M}+1]^{+}, 100\right)$.

4-丙基-5-[4-(三氟甲氧基)苯基]嘧啶-2-胺(6c)：12.0 $\mathrm{mg}$, 产率 40\%, LC-MS 分析纯度(HPLC: 80.2\%, ELSD: $100 \%)$, m.p. 590.20, 分子式 $\mathrm{C}_{14} \mathrm{H}_{14} \mathrm{~F}_{3} \mathrm{~N}_{3} \mathrm{O} .{ }^{1} \mathrm{H}$ NMR $\left(\mathrm{CD}_{3} \mathrm{OD}, 400 \mathrm{MHz}\right) \delta: 0.90\left(\mathrm{t}, J=6 \mathrm{~Hz}, 3 \mathrm{H}, \mathrm{CH}_{3}\right), 1.66 \sim$ $1.71\left(\mathrm{~m}, 2 \mathrm{H}, \mathrm{CH}_{2}\right), 2.44\left(\mathrm{t}, J=6 \mathrm{~Hz}, 2 \mathrm{H}, \mathrm{CH}_{2}\right), 7.05$ (d, $J=8 \mathrm{~Hz}, 2 \mathrm{H}$, benzene), $7.68(\mathrm{~d}, J=8 \mathrm{~Hz}, 2 \mathrm{H}$, benzene), 8.95 (s, $1 \mathrm{H}$, pyrimidine); ${ }^{13} \mathrm{C}$ NMR (DMSO- $d_{6}, 100 \mathrm{MHz}$ ) $\delta: 169.60,161.08,149.88,148.72,128.90,126.43,126.36$, 124.37, 120.66, 115.30, 38.60, 23.04, 12.58; ESI-MS $m / z$ (\%): $298\left([\mathrm{M}+1]^{+}, 100\right)$.

4-(2-甲基丁基)-5-[4-(三氟甲基)苯基]嘧啶-2-胺 (6d): $15.5 \mathrm{mg}$, 产率 50\%, LC-MS 分析纯度 $96.3 \%$, m.p. 575.51, 分子式 $\mathrm{C}_{16} \mathrm{H}_{18} \mathrm{~F}_{3} \mathrm{~N}_{3},{ }^{1} \mathrm{H}$ NMR $\left(\mathrm{CD}_{3} \mathrm{OD}, 400 \mathrm{MHz}\right)$ $\delta: 0.90\left(\mathrm{t}, J=6 \mathrm{~Hz}, 3 \mathrm{H}, \mathrm{CH}_{3}\right), 1.02(\mathrm{~d}, J=10 \mathrm{~Hz}, 3 \mathrm{H}$, $\left.\mathrm{CH}_{3}\right), 1.55 \sim 1.60\left(\mathrm{~m}, 2 \mathrm{H}, \mathrm{CH}_{2}\right), 2.04 \sim 2.10(\mathrm{~m}, 1 \mathrm{H}, \mathrm{CH})$, $2.51\left(\mathrm{~d}, J=8 \mathrm{~Hz}, 2 \mathrm{H}, \mathrm{CH}_{2}\right), 7.35(\mathrm{~d}, J=8 \mathrm{~Hz}, 2 \mathrm{H}$, benzene), 7.68 (d, $J=8 \mathrm{~Hz}, 2 \mathrm{H}$, benzene), $8.92(\mathrm{~s}, 1 \mathrm{H}$, pyrimidine); ${ }^{13} \mathrm{C}$ NMR (DMSO- $d_{6}$ ) $\delta: 167.70,159.86$, $149.89,137.10,132.35,127.80,124.20,123.68,119.30$, $40.05,35.87,31.42,21.56,10.54$; ESI-MS $m / z$ (\%): 310 $\left([\mathrm{M}+1]^{+}, 100\right)$.

4-异丁基-5-[4-(三氟甲基)苯基]嘧啶-2-胺(6e)：20.1 $\mathrm{mg}$, 产率 65\%, LC-MS 分析纯度 $97.1 \%$, m.p. 564.24, 分 子式 $\mathrm{C}_{15} \mathrm{H}_{16} \mathrm{~F}_{3} \mathrm{~N}_{3} .{ }^{1} \mathrm{H} \mathrm{NMR}\left(\mathrm{CD}_{3} \mathrm{OD}, 400 \mathrm{MHz}\right) \delta: 0.91(\mathrm{~d}$, $\left.J=5 \mathrm{~Hz}, 6 \mathrm{H}, \mathrm{CH}_{3}\right), 1.82 \sim 1.86(\mathrm{~m}, 1 \mathrm{H}, \mathrm{CH}), 2.51(\mathrm{~d}, J=8$ $\left.\mathrm{Hz}, 2 \mathrm{H}, \mathrm{CH}_{2}\right), 7.39$ (d, $J=8 \mathrm{~Hz}, 2 \mathrm{H}$, benzene), 7.68 (d, $J=$ $8 \mathrm{~Hz}, 2 \mathrm{H}$, benzene), 8.95 (s, $1 \mathrm{H}$, pyrimidine); ${ }^{13} \mathrm{C}$ NMR $\left(\right.$ DMSO- $\left._{6}\right) \delta: 165.58,158.97,150.83,137.24,132.43$, $127.45,125.62,123.35,118.35,41.67,30.84,21.56$; ESIMS $m / z$ (\%): $296\left([\mathrm{M}+1]^{+}, 100\right)$. 
4-丙基-5-[4-(三氟甲基)苯基]嘧啶-2-胺(6f)：23.7 $\mathrm{mg}$, 产率 35\%, LC-MS 分析纯度 83.8\%, m.p. 567.97, 分 子式 $\mathrm{C}_{14} \mathrm{H}_{14} \mathrm{~F}_{3} \mathrm{~N}_{3} .{ }^{1} \mathrm{H}$ NMR $\left(\mathrm{CD}_{3} \mathrm{OD}, 400 \mathrm{MHz}\right) \delta: 0.93(\mathrm{t}$, $\left.J=8 \mathrm{~Hz}, 3 \mathrm{H}, \mathrm{CH}_{3}\right), 1.66 \sim 1.70\left(\mathrm{~m}, 2 \mathrm{H}, \mathrm{CH}_{2}\right), 2.44(\mathrm{t}, J=$ $8 \mathrm{~Hz}, 2 \mathrm{H}, \mathrm{CH}_{2}$ ), 7.35 (d, $J=8 \mathrm{~Hz}, 2 \mathrm{H}$, benzene), 7.68 (d, $J=8 \mathrm{~Hz}, 2 \mathrm{H}$, benzene), 8.92 (s, $1 \mathrm{H}$, pyrimidine); ${ }^{13} \mathrm{C}$ NMR $\left(\right.$ DMSO- $_{6}$ ) $\delta$ : $167.41,159.77,149.30,136.07,131.80$, $127.53,125.66,123.32,119.05,38.60,24.00,12.81$; ESI-MS $m / z(\%): 282\left([\mathrm{M}+1]^{+}, 100\right)$.

5-(4-氯苯基)-4-(2-甲基丁基)嘧啶-2-胺 (6g)：25.0 $\mathrm{mg}$, 产率 48\%, LC-MS 分析纯度 90.9\%, m.p. 589.97, 分 子式 $\mathrm{C}_{15} \mathrm{H}_{18} \mathrm{ClN}_{3},{ }^{1} \mathrm{H}$ NMR $\left(\mathrm{CD}_{3} \mathrm{OD}, 400 \mathrm{MHz}\right) \delta: 0.90(\mathrm{t}$, $\left.J=6 \mathrm{~Hz}, 3 \mathrm{H}, \mathrm{CH}_{3}\right), 0.96\left(\mathrm{~d}, J=10 \mathrm{~Hz}, 3 \mathrm{H}, \mathrm{CH}_{3}\right), 1.55 \sim$ $1.60\left(\mathrm{~m}, 2 \mathrm{H}, \mathrm{CH}_{2}\right), 2.04 \sim 2.09(\mathrm{~m}, 1 \mathrm{H}, \mathrm{CH}), 2.51$ (d, $J=$ $\left.12 \mathrm{~Hz}, 2 \mathrm{H}, \mathrm{CH}_{2}\right), 7.43$ (d, $J=8 \mathrm{~Hz}, 2 \mathrm{H}$, benzene), 7.73 (d, $J=8 \mathrm{~Hz}, 2 \mathrm{H}$, benzene), 8.90 (s, $1 \mathrm{H}$, pyrimidine); ${ }^{13} \mathrm{C}$ NMR (DMSO- $d_{6}$ ) $\delta$ : $167.51,159.82,149.16,135.10,132.30$, 129.00, 128.57, 119.27, 40.02, 36.07, 30.42, 21.42, 10.51; ESI-MS $m / z(\%): 276\left([\mathrm{M}+1]^{+}, 100\right), 278\left([\mathrm{M}+3]^{+}\right.$, 34.4).

5-(4-氯苯基)-4-异丁基嘧啶-2-胺(6h): $18.3 \mathrm{mg}$, 产 率 70\%, LC-MS 分析纯度 $100 \%$, m.p. 578.70, 分子式 $\mathrm{C}_{14} \mathrm{H}_{16} \mathrm{ClN}_{3} .{ }^{1} \mathrm{H}$ NMR $\left(\mathrm{CD}_{3} \mathrm{OD}, 400 \mathrm{MHz}\right) \delta: 0.91$ (d, $J=6$ $\left.\mathrm{Hz}, 6 \mathrm{H}, \mathrm{CH}_{3}\right), 1.82 \sim 1.87(\mathrm{~m}, 1 \mathrm{H}, \mathrm{CH}), 2.51(\mathrm{~d}, J=12 \mathrm{~Hz}$, 2H, $\mathrm{CH}_{2}$ ), 7.51 (d, $J=8 \mathrm{~Hz}, 2 \mathrm{H}$, benzene), 7.73 (d, $J=8$ $\mathrm{Hz}, 2 \mathrm{H}$, benzene), 8.95 (s, $1 \mathrm{H}$, pyrimidine); ${ }^{13} \mathrm{C}$ NMR (DMSO- $d_{6}$ ) $\delta$ : $167.57,159.60,149.54,135.13,130.35$, 129.13, 128.60, 119.77, 42.22, 28.76, 27.46; ESI-MS $m / z$ (\%): $262\left([\mathrm{M}+1]^{+}, 100\right), 264\left([\mathrm{M}+3]^{+}, 35.1\right)$.

5-(4-氯苯基)-4-丙基嘧啶-2-胺(6i): $14.5 \mathrm{mg}$, 产率 $58 \%$, LC-MS 分析纯度 $94.8 \%$, m.p. 582.43, 分子式 $\mathrm{C}_{13} \mathrm{H}_{14} \mathrm{ClN}_{3} .{ }^{1} \mathrm{H}$ NMR ( $\left.\mathrm{CD}_{3} \mathrm{OD}, 400 \mathrm{MHz}\right) \delta: 0.93$ (t, $J=5$ $\left.\mathrm{Hz}, 3 \mathrm{H}, \mathrm{CH}_{3}\right), 1.66 \sim 1.70\left(\mathrm{~m}, 2 \mathrm{H}, \mathrm{CH}_{2}\right), 2.44(\mathrm{t}, J=10 \mathrm{~Hz}$, $\left.2 \mathrm{H}, \mathrm{CH}_{2}\right), 7.5(\mathrm{~d}, J=8 \mathrm{~Hz}, 2 \mathrm{H}$, benzene), $7.73(\mathrm{~d}, J=8 \mathrm{~Hz}$, $2 \mathrm{H}$, benzene), 8.92 (s, $1 \mathrm{H}$, pyrimidine); ${ }^{13} \mathrm{C}$ NMR (DMSO$\left.d_{6}\right) \delta: 165.67,158.82,150.92,136.57,132.75,130.92$, 128.47, 121.62, 37.67, 22.64, 15.51; ESI-MS $m / z$ (\%): 248 $\left([\mathrm{M}+1]^{+}, 100\right), 250\left([\mathrm{M}+3]^{+}, 33.8\right)$.

5-(4-氯-2-氟苯基)-4-(2-甲基丁基)嘧啶-2-胺 (6j): $13.2 \mathrm{mg}$, 产率 45\%, LC-MS 分析纯度(HPLC: 90.0\%, ELSD: 96.9\%), m.p. 603.08, 分子式 $\mathrm{C}_{15} \mathrm{H}_{17} \mathrm{ClFN}_{3} .{ }^{1} \mathrm{H}$ NMR $\left(\mathrm{CD}_{3} \mathrm{OD}, 400 \mathrm{MHz}\right) \delta: 0.90\left(\mathrm{t}, J=6 \mathrm{~Hz}, 3 \mathrm{H}, \mathrm{CH}_{3}\right)$, $0.96\left(\mathrm{~d}, J=10 \mathrm{~Hz}, 3 \mathrm{H}, \mathrm{CH}_{3}\right), 1.55 \sim 1.60\left(\mathrm{~m}, 2 \mathrm{H}, \mathrm{CH}_{2}\right)$, $2.04 \sim 2.10(\mathrm{~m}, 1 \mathrm{H}, \mathrm{CH}), 2.51\left(\mathrm{~d}, J=12 \mathrm{~Hz}, 2 \mathrm{H}, \mathrm{CH}_{2}\right)$, 7.46 (s, $1 \mathrm{H}$, benzene), 7.50 (d, $J=8 \mathrm{~Hz}, 1 \mathrm{H}$, benzene),
7.53 (d, $J=8 \mathrm{~Hz}, 1 \mathrm{H}$, benzene), 8.90 (s, 1H, pyrimidine); ${ }^{13} \mathrm{C}$ NMR (DMSO- $d_{6}$ ) $\delta: 165.75,163.75,161.04,150.27$, $136.81,132.55,125.76,121.50,120.36,117.20,40.02$, $36.07,30.42,21.42,10.51$; ESI-MS $m / z$ (\%): 294 ([M+ $\left.1]^{+}, 100\right), 296\left([\mathrm{M}+3]^{+}, 34.6\right)$.

5-(4-氯-2-氟苯基)-4-异丁基嘧啶-2-胺(6k): $17.1 \mathrm{mg}$, 产率 60\%, LC-MS 分析纯度 $97.2 \%$, m.p. 591.81, 分子式 $\mathrm{C}_{14} \mathrm{H}_{15} \mathrm{ClFN}_{3} .{ }^{1} \mathrm{H}$ NMR $\left(\mathrm{CD}_{3} \mathrm{OD}, 400 \mathrm{MHz}\right) \delta: 0.91$ (d, $J=$ $\left.6 \mathrm{~Hz}, 6 \mathrm{H}, \mathrm{CH}_{3}\right), 1.82 \sim 1.85(\mathrm{~m}, 1 \mathrm{H}, \mathrm{CH}), 2.51(\mathrm{~d}, J=10$ $\left.\mathrm{Hz}, 2 \mathrm{H}, \mathrm{CH}_{2}\right), 7.46(\mathrm{~s}, 1 \mathrm{H}$, benzene), $7.50(\mathrm{~d}, J=8 \mathrm{~Hz}, 1 \mathrm{H}$, benzene), 7.54 (d, $J=8 \mathrm{~Hz}, 1 \mathrm{H}$, benzene), $8.93(\mathrm{~s}, 1 \mathrm{H}$, pyrimidine); ${ }^{13} \mathrm{C}$ NMR (DMSO- $d_{6}$ ) $\delta: 165.77,163.82$, $160.26,150.92,136.57,132.75,125.76,121.54,120.36$, 118.35, 40.65, 29.74, 22.91; ESI-MS $m / z$ (\%): 280 ([M+ $\left.1]^{+}, 100\right), 282\left([\mathrm{M}+3]^{+}, 32.6\right)$.

5-(4-氯-2-氟苯基)-4-丙基嘧啶-2-胺(61): $14.6 \mathrm{mg}$, 产率 54\%, LC-MS 分析纯度 (HPLC: $88.4 \%$, ELSD: 96.9\%), m.p. 595.54, 分子式 $\mathrm{C}_{13} \mathrm{H}_{13} \mathrm{ClFN}_{3} .{ }^{1} \mathrm{H}$ NMR $\left(\mathrm{CD}_{3} \mathrm{OD}, 400 \mathrm{MHz}\right) \delta: 0.90\left(\mathrm{t}, J=6 \mathrm{~Hz}, 3 \mathrm{H}, \mathrm{CH}_{3}\right), 1.66 \sim$ $1.71\left(\mathrm{~m}, 2 \mathrm{H}, \mathrm{CH}_{2}\right), 2.44\left(\mathrm{t}, J=10 \mathrm{~Hz}, 2 \mathrm{H}, \mathrm{CH}_{2}\right), 7.46(\mathrm{~s}$, $1 \mathrm{H}$, benzene), 7.50 (d, $J=8 \mathrm{~Hz}, 1 \mathrm{H}$, benzene), 7.53 (d, $J=$ $8 \mathrm{~Hz}, 1 \mathrm{H}$, benzene), 8.92 (s, $1 \mathrm{H}$, pyrimidine); ${ }^{13} \mathrm{C}$ NMR $\left(\right.$ DMSO- $_{6}$ ) $\delta: 167.30,164.00,160.36,150.79,136.60$, $132.81,125.50,121.34,120.23,119.07,38.68,23.34$, 12.65; ESI-MS $m / z(\%): 266\left([\mathrm{M}+1]^{+}, 100\right), 268$ ([M+ $\left.3]^{+}, 33.6\right)$.

\section{2 结果与讨论}

本课题组研究 2-氨基 4,5-二烃基取代嘧啶类衍生物 合成的新方法. 合成烷基芳香酮 4 这一步反应，根据文 献[9 11]报道的条件, 首先反应过程要求避光; 其次溶 剂的选择, 本课题组选用过四氢呋喃、甲醇和乙醇分别 作为溶剂, 但前两者溶剂经实验后检测, 副反应比较多, 而所需的目标产物非常少. 故我们最终选用乙醇钠为 碱，在乙醇中回流反应，即可得到 12 个烷基芳香酮 $\mathbf{4}$, 实验结果与文献[10]报道相符.

中间体 5 的合成，烷基芳香酮 $\mathbf{4}$ 与 DMF-二甲基缩 醛溶液的反应通常是在有机溶剂中进行 ${ }^{[12,13]}$, 本文的中 间体 5 的合成不另加溶剂, 将烷基芳香酮 4 与过量的 DMF-二甲基缩醛进行加热回流反应，过量的 DMF-二 甲基缩醛兼作反应溶剂 ${ }^{[14]}$. 当烷基芳香酮 4 与过量的 DMF-二甲基缩醛的物质的量的比在 $1: 1.4 \sim 1: 1.5$ 时, 各产物的收率最好.

本合成路线以廉价简单的作为原料，避免使用剧毒 性化学药品, 合成操作简单, 便于实验操作, 经四步反 
应，每步的中间体后处理方便. 且用此方法合成嘧啶类 化合物，目前国内尚未见报道. 这些化合物的进一步结 构修饰以及药物活性篎选工作还正在研究中.

\section{References}

[1] Wu, Q.; Song, A.-B.; Jin, L.-H.; Hu, D.-Y. Chin. J. Org. Chem. 2009, 29(3), 365 (in Chinese). (吴琴, 宋宝安, 金林红, 胡德禹, 有机化学, 2009, 29(3), 365.)

[2] Wang, Z.; Xu, Y.; Dong, W.; Liang, P.; Yang, X.-L. Chin. J. Org. Chem. 2011, 31(4), 567 (in Chinese) (王振, 徐炎, 董玮, 梁沛, 杨新玲, 有机化学, 2011, 31(4), 567.)

[3] Li, Y.-X.; Lin, L.; Chen, Q. Chin. J. Org. Chem. 2011, 31(3), 306 (in Chinese). (李元祥, 林龙, 陈琼, 有机化学, 2011, 31(3), 306.)

[4] Bridges, A.-J. Chem. Rev. 2001, 101, 2541.

[5] Pranab, K.; Mahata U.-K.; Syam, K.-V.; Sriram, H.-I.; Junjappa, H.
Tetrahedron 2003, 59, 2631.

[6] Deshmukh, M.-B.; Salunkhe, S.-M.; Patil, D.-R.; Anbhule, P.-V. Eur. J. Med. Chem. 2009, 44, 2651.

[7] Takahashi, S.; Togo, H. Heterocycles 2010, 1(82), 593.

[8] Aggarwal, V.-K.; Vicente, J.-D.; Pelotier, B.; Holmes, I.-P.; Bonnert, R.-V. Tetrahedron Lett. 2000, 41, 10327.

[9] Fulton, J.-R.; Aggarwal, V.-K.; Vicente, J.-D. Eur. J. Org. Chem. 2005, 1479.

[10] Angle, S.-R.; Neitzel, M.-L. J. Org. Chem. 2000, 65, 6458.

[11] Angle, S.-R.; Neitzel, M.-L. J. Org. Chem. 1999, 64, 8754.

[12] Kil, K.-E; Ding, Y.-S; Lin, K.-S. Nucl. Med. Biol. 2007, 34, 153.

[13] Plate, R.; Plaum, M.-J.-M.; Deboer, T. Bioorg. Med.Chem. 1996, 4, 227.

[14] Chen, Q.-F.; Zhang, H.-D.; Gong, S.-C. Chin. J. Appl. Chem. 2011, 4, 382 (in Chinese).

(陈启凡，张慧东，宫胜臣，应用化学，2011，4, 382.) 